\title{
Entrelacs
}

ENTRELACS Cinéma et audiovisuel

11 | 2014

La Voix

\section{Chant contre champ, Chantal Akerman : Chanter, se libérer}

Emma Dusong

\section{(2) OpenEdition}

Journals

Édition électronique

URL : http://journals.openedition.org/entrelacs/1020

DOI : 10.4000 /entrelacs. 1020

ISSN : 2261-5482

Éditeur

Éditions Téraèdre

Référence électronique

Emma Dusong, "Chant contre champ, Chantal Akerman : Chanter, se libérer », Entrelacs [En ligne],

11 | 2014, mis en ligne le 31 décembre 2014, consulté le 30 avril 2019. URL : http://

journals.openedition.org/entrelacs/1020; DOI : 10.4000/entrelacs.1020

Ce document a été généré automatiquement le 30 avril 2019

Tous droits réservés 


\title{
Chant contre champ, Chantal Akerman : Chanter, se libérer
}

\author{
Emma Dusong
}

Comment faire pour vivre? Comment faire pour vivre malgré tout? Comment faire pour vivre avec le passé ? Comment faire pour vivre au présent? Comment vivre tout simplement? Il y a cette mémoire, que l'on peut sentir, que l'on respire. Les camps, la guerre, la survivance, l'héritage de cette survivance. Et puis il y a le maintenant. Car il faut vivre n'est-ce pas?

2 Peut-être que les films de Chantal Akerman parlent (souvent) de cela : du vivre malgré tout, entre vie et survivance. De vivre en décalé. De vivre à la folie. De vivre soi. De vivre sa vérité, de provoquer sa vérité, ses désirs, son vivre. Comment vivre sa voie? Comment imaginer l'émancipation, la transgression, le flux, la mobilité ? Comment sortir de l'empêchement? Il y a l'image, les images, les cadres, les cadres clos dont on a du mal à sortir. Et puis il y a la voix, la parole, la litanie, la polyphonie, et enfin le chant.

3 Il faut prendre souffle pour chanter. Dans le prolongement de la respiration, chanter apparaît comme un médium vivant, alliant la corporalité aux mots, il permet de s'exprimer tout entier. Loin des chansons courtoises et des chants des sirènes, les chants de Chantal Akerman agissent en fin de film, en fin d'histoire ${ }^{1}$. Ils ne sont pas des points de départ pour engager la rencontre mais une ouverture finale, un dénouement possible pour les personnages. Nous avons été témoin d'une transition et maintenant qu'ils chantent, ils s'affranchissent.

\section{Je, tu, il, elle et Saute ma ville}

Dans son film Je, tu, il, elle $e^{2}$ la cinéaste choisie la comptine Nous n'irons plus aux bois. De structure simple, souvent chantée par les enfants, une comptine est répétitive et joue un rôle important dans l'apprentissage du langage vocal et parfois gestuel. Les paroles aux airs ingénus peuvent avoir un caractère historique, politique ou libertin. C'est une phrase chantée ou parlée qui donne un rôle distinctif lors d'un jeu. Am, stram, gram, par exemple, 
sélectionne les participants. Ici Nous n'irons plus aux bois engage les joueurs dans la danse. Si la forme choisie se réfère à l'enfance, c'est pour mieux s'en mettre à distance. Elle opère en décalage, " décal-âge ». Revenir à l'enfance pour mieux souligner la rupture et passer dans le monde des adultes, dans ce monde où l'on agit plutôt que d'observer. Mais Chantal Akerman n'est pas dupe : «J'ai une vie occupée et j'ai l'air d'être définitivement passée du côté des adultes avec le sourire, mais je crois qu'en chacun de nous, il reste une résonance de ce hurlement d'un moment, que l'on a étouffé pour jouer le jeu de la société $»^{3}$. La comptine enfantine associée à la sexualité adulte devient alors une brèche dans un temps linéaire, une transgression où tous les âges sont à portée. Au passage vers le monde adulte, la réalisatrice revient aux sources de l'enfance pour marquer le saut. Il ne s'agit pas de se rebeller, mais de revivre l'origine de soi. Je, tu, il, elle prend à bras-lecorps l'éveil des sens et de la sexualité en trois espaces-temps. Ces étapes libèrent Julie du trouble qui la cloître dans sa chambre. Dans la première partie, la jeune femme, interprétée par Chantal Akerman, écrit des lettres et mange du sucre en grande quantité. Sa voix décrit sa situation. Le «Je » du titre suggère Julie à son bureau, dans sa chambre rangée. Le «tu » apparaît quand elle se déshabille, vide sa chambre et se retrouve nue face à elle-même. Lorsqu'elle sort de chez elle et se déplace en auto-stop, elle rencontre « il » (joué par Niels Arestrup), un camionneur, qui lui parle de sa relation aux femmes, du désir et de la fuite du désir. Enfin, elle retrouve « elle » (jouée par Claire Wauthion), la femme convoitée, qui la repousse dans un premier temps puis l'accepte dans son lit. Au matin, en plan fixe et frontal, elle se lève, sort du champ à gauche pour ouvrir les rideaux et éclairer ainsi son amie puis traverse le cadre en tenant ses habits pour sortir cette fois du champ à droite. Commence ensuite la comptine qui se poursuit sur le générique.

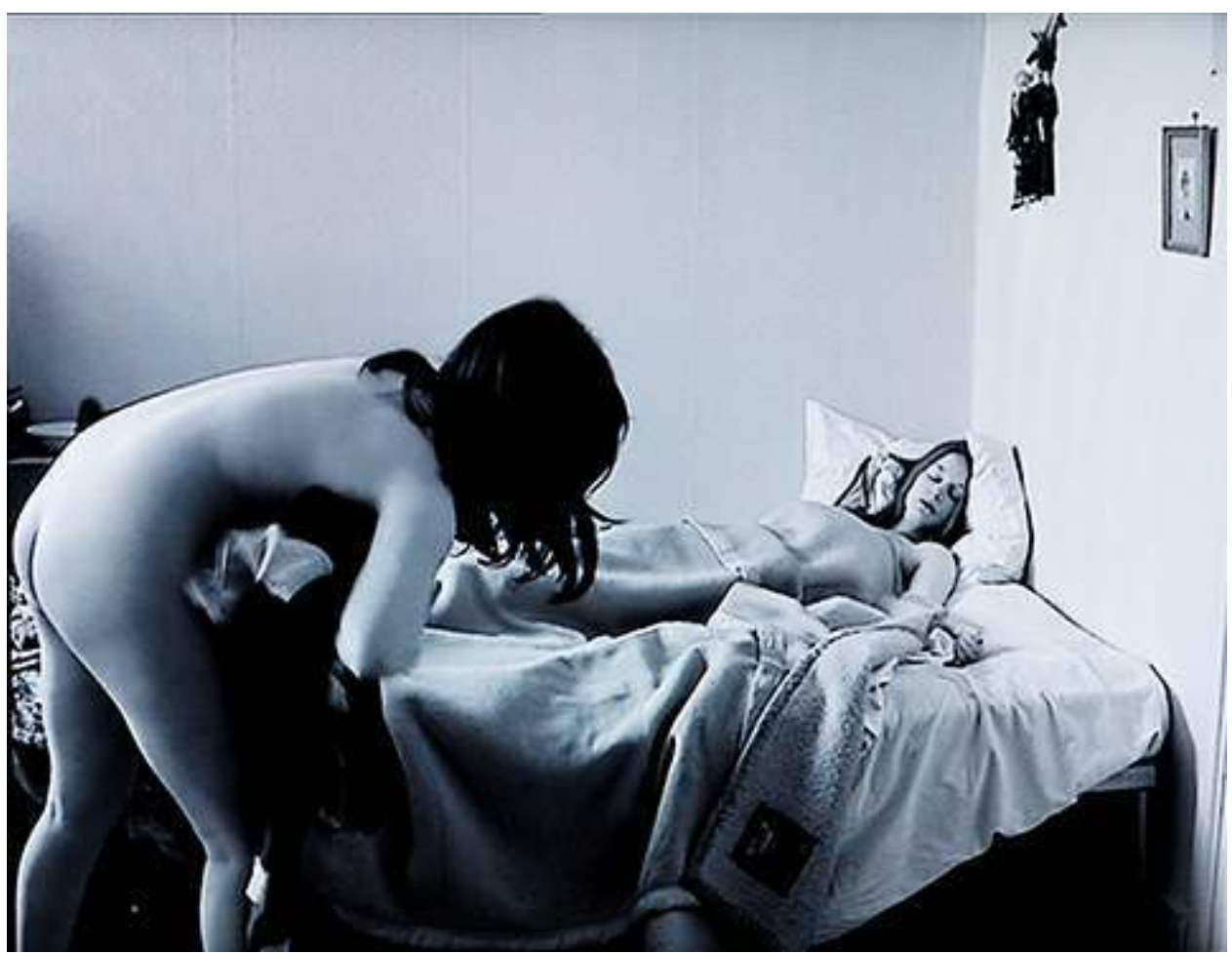

Chantal Akerman, Je, tu, il, elle, long-métrage, 90 minutes, 1974.

La voix de Julie, presque sans âge, presque sans genre, moins habile, légèrement plus basse en volume que celle de « elle » suit les consignes chantées et répète les paroles de la comptine. Ce sont des enfants qui commencent et entraînent le spectateur: "Nous 
n'irons plus aux bois, les lauriers sont coupés » puis l'amie chante d'une voix féminine et affirmée: "la belle que voilà les aura ramassés ». Les paroles précisent le levier du changement. C'est grâce à la rencontre de l'autre, de « la belle », de " elle » que Julie peut se dépasser. "Voyez comme on danse » indique un chemin à suivre. Julie accompagne cette fois les enfants et chante: "Sautez, dansez, embrassez qui vous voudrez » qui souligne le libre choix de la personne convoitée, au-delà des normes de la société. « Nous n'irons plus au bois" suggère l'espace où l'on peut se cacher, le "bois profond et maternel $»^{4}$. Il est question de prendre un risque, de quitter cet univers protégé. La relation à la mère est un thème récurrent chez la cinéaste. Dans News from home (1976), elle lit les lettres reçues, souvent plaintives et inquiètes, de sa mère sur des plans de New York. La mère n'apparaît pas à l'image, mais sa présence est omniprésente, même envahissante, par la lecture à haute voix de sa fille. La voix semble fixer le regard de ces longs plans. On retrouve la mère dans les films Les rendez-vous d'Anna (1978), Aujourd'hui dis-moi (1980) où sa mère parle en voix over de ses grands-mères, Demain, on déménage (2004) et surtout dans son livre Ma mère rit ${ }^{5}$ (2013) où la cinéaste alors auteure écrit sa peur de voir sa mère mourir et l'angoisse de la perdre.

6 On entend ensuite: " les lauriers sont coupés». C'est une période révolue que Julie accepte. L'alternance continue entre les deux femmes: «La belle que voilà les aura ramassés, entrez dans la danse, voyez comme on danse. » Julie prend confiance en ellemême et agit. Elle donne vie à son désir profond. «Sautez, dansez, embrassez qui vous voudrez » témoigne d'une prise de liberté. Puis elles reprennent à deux l'ensemble de la comptine. Chanter soutient Julie et l'accompagne dans sa difficulté de faire. Dans Le journal d'une paresseuse ${ }^{6}$, Chantal Akerman s'encourage également en chantant : « Lève-toi, paresseuse, habille-toi » Ces actions ordinaires de tous les jours reviennent dans Lettre d'une cinéaste : Chantal Akerman ${ }^{7}$ où elle explique: «Faire du cinéma, c'est se lever, s'habiller, manger », ce qu'elle reprend à nouveau dans son livre en $2004:$ « Pour faire du cinéma, faut se lever. Je me lève. Pour faire du cinéma, faut s'habiller, je m'habille. Pour faire du cinéma, faut être debout, je suis debout $»^{8}$. Taches qui semble-t-il ne sont pas si simples. La répétition des formules suggère le rythme à prendre, face à l'étendue des journées. Proche d'une prière juive et d'un rituel, les phrases deviennent des mélopées qui lient le chant au faire. Elle raconte d'ailleurs, à propos de Ma mère rit, comment le ton et le rythme de l'écriture vocale puise dans ses premières expériences de l'oralité à la stiebel, la petite synagogue où son grand-père l'emmenait ${ }^{9}$. Le rythme des phrases, proche d'une litanie, amorce la mélodie. Sur le plan étymologique, le mot « mélopée » se compose de mélos soit chant ou air et de poëô soit faire. La voix se place dans le prolongement du parler, comme si une fine mélodie était venue s'accrocher aux mots pour les faire danser et leur permettre d'agir. Le glissement musical permet alors de quitter le ressassement.

7 Le chant Nous n'irons plus aux bois rejoue le principe des pronoms personnels du titre et de la rencontre avec chaque personnage. En effet, traditionnellement, à chaque couplet un enfant entre dans la ronde et remplace l'enfant précédent jusqu'à ce que chaque joueur ait participé. Ainsi l'air de rien la comptine reprend l'ensemble du film, de l'isolement de Julie à la rencontre de l'homme puis de la femme, dans une forme courte chantée qui ouvre une perspective plus épanouissante au personnage. Cette répétition narrative sous forme vocale rappelle celle de Cléo de 5 à $7^{10}$ d'Agnès Varda en sens inverse. Au début du film, les éléments essentiels du scénario sont donnés. Le long-métrage ouvre sur une séance de tarot où Cléo espère retrouver espoir face à sa crainte du cancer. Tous les dires de la voyante se réaliseront dans la suite du film. À l'image des génériques de Saul Bass 
qui donne la clé de lecture des films, Agnès Varda donne le canevas narratif. Pourtant la fin du film déjoue l'anticipation du spectateur lorsque cléo se dit heureuse quand elle apprend sa maladie. On se rappelle alors des paroles de la vielle femme: «Cette carte n'est pas forcément la mort, les pieds et les mains sont couverts de chair, c'est une transformation profonde de tout votre être ». Le temps du film et de l'histoire ${ }^{11}$ ont permis à Cléo de voir la vie différemment, elle s'est lentement ouverte au monde, aux autres jusqu'à échanger avec un inconnu. Cette métamorphose lui permet de devenir maître de son récit. En utilisant inversement une forme répétée en fin de film, Chantal Akerman invite Julie, non pas à sortir d'une histoire préétablie, mais d'un cadre prédéfini grâce à la mobilité de la voix chantée. Dans Cinéma 2, L'image-temps, Gilles Deleuze reprend la question d'Alain Philippon posée à Chantal Akerman et écrit : «S'il est possible d'éviter l'excès de stylisation qui tend, malgré tout, à renfermer film et personnage, c'est le problème que Chantal Akerman pose elle-même $»^{12}$. Avec la voix chantée libérée de toute dimension technique, la cinéaste parvient à échapper à la "perfection $»^{13}$ de ses cadrages.

8 La comptine Nous n'irons plus au bois date probablement du moyen âge. La mélodie provient des premières notes du Kyrie de la messe De Angelis et les paroles sont le plus souvent attribuées à la marquise de Pompadour, amante du Roi Louis XV. Certaines interprétations, invérifiables aujourd'hui, affirment une référence à des rencontres interdites et extraconjugales. Les lauriers suggéreraient une récolte impossible au sein du mariage ou bien renverraient vers les branches d'églantier des entrées des «bordels ». D'autres historiens font un rapport avec les bosquets du parc de Versailles qui furent coupés car les couples illégitimes s'y retrouvaient. Toutes ces analyses témoignent d'un désir interdit et de la volonté de le vivre malgré tout. Chantal Akerman, venant d'une famille religieuse traditionnelle, n'a pas voulu que le film sorte en Belgique car selon elle, la scène d'amour entre les deux femmes aurait tué son père ${ }^{14}$. Dans le film, Julie vit son attirance jusqu'au bout, les gestes d'amour ressemblent à des gestes de lutte. Ils témoignent d'un excès des désirs agités. On entend les prémices d'un chant, un fredonnement de plaisir qui annonce la comptine finale. Dans son premier court-métrage, Saute ma ville ${ }^{15}$, réalisé à dix-huit ans, Chantal Akerman travaille déjà le fredonnement, parfois grogné, d'un bouillonnement intérieur. Ici le chant, dans une pulsion de mort, renvoi au dernier soupir telle une dernière expiration avant le suicide. Il est aussi élan de vie, ultime expression et retour à l'origine du langage. On passe du signifié au signifiant. La voix quitte la codification des mots pour dire par elle-même. Mladen Dolar écrit à propos de la voix :

" On peut sans doute attribuer du sens à toutes sortes de sons, pourtant ils semblent en être dépourvus en eux-mêmes, alors que la voix a une connexion intime avec lui, c'est un son qui semble pourvu de la volonté de dire quelque chose, avec une intentionnalité propre, elle ouvre à la supposition d'une intériorité qui s'exprime $»^{16}$.

Dans Saute ma ville, la voix de Chantal Akerman qui chante, chantonne, soupire, piaffe, scande, ressasse, murmure, crie, nous énonce son agitation, sa démesure, sa fièvre et son excitation, sa boulimie, sa rébellion, son désespoir latent, son irruption... Au bout des mots, elle use de la voix pour ouvrir l'écoute, provoquer les sensations. Le chant, dans cette cuisine où la jeune femme a décidé d'en finir, anticipe l'explosion finale. Chantal Akerman convoque ainsi son premier choc cinématographique: Pierrot le fou ${ }^{17}$ où le personnage s'explose la tête en fin de film après avoir répété merde, merde comprenant que ce n'est plus un jeu mais une réalité. Faire tout péter pour se libérer. La cinéaste, née après-guerre en 1950 en Belgique, raconte comment sa mère, survivante des camps de 
concentration, n'a jamais parlé de son traumatisme à ses enfants, transmis pourtant dans la chair ${ }^{18}$. Le traumatisme légué à la génération suivante ne semble pas saisissable car il n'a pas été vécu de façon directe. Le passé est toujours présent et la possibilité de vivre sereinement est difficile. Dans Saute ma ville, la jeune femme utilise le gaz pour tout exploser. Mais dimension autobiographique mise de côté, la douleur agitée, la violence contenue, le désir de vivre tambourine de l'intérieur les personnages. Seule émission intelligible de son implosion chantée, le mot « danse " revient à deux reprises comme une mise en abîme de cette chorégraphie du corps et de la voix. Se débattre du cadre est également présent dans $\mathrm{Je}$, tu, il, elle, où elle reste enfermée toute la première partie du film puis retrouve le dehors qu'elle observait de sa chambre au bord de l'image. On passe de l'explosion finale dans Saute ma ville, au "chanter» d'une comptine. Les envies s'articulent et associent chant et accomplissement.

Boris Lehman remarque cependant : «Rien n'a fondamentalement changé dans le destin de ses héroïnes, elles finissent toujours par partir avec leur valise. Jamais de vrai happy end. Jamais de vraie vie nouvelle qui point à l'horizon. Au contraire, l'horizon est bouché, la route est sans issue et le personnage seul, désemparé (air connu), criminel, au bord du suicide. Elle l'a fait, elle est donc folle. Elle l'a fait parce qu'il le fallait, tout le monde doit y passer un jour $»^{19}$. Certes la fin ne correspond pas à un happy end normé avec la formation d'un couple joyeux et épanoui, mais le chant témoigne d'une ouverture pour Julie. Elle a pu avancer et quitter son malaise. Chanter apparaît comme le levier d'une autonomie des trois actes classiques de la tragédie. Reste cependant, que la répétition en jeu: c'est l'amie qui chante et Julie qui répète, souligne la notion du devoir d'une rencontre à mener et témoigne d'une ambivalence face au plaisir.

\section{Demain, on déménage}

11 Trente ans plus tard, Chantal Akerman réalise Demain, on déménage ${ }^{20}$. Cette fois son personnage Charlotte (interprétée par Sylvie Testud) doit écrire un roman érotique et manque d'inspiration, ignorant le désir. Elle décide d'écouter ses voisins et d'observer les couples qui se forment et se séparent autour d'elle. Elle achève son livre grâce aux rencontres qui traversent son appartement et à une chambre où elle et son amie se relaient pour écrire. La femme enceinte apparue plus tôt la rejoint à la fin du film. Elle lui annonce : «Je vais vivre avec vous » puis diffuse une chanson chantée par Charlotte et accompagnée d'un piano. La femme à l'enfant danse et Charlotte lui fait un thé. Les rôles à l'œuvre dans $\mathrm{Je}$, tu, il, elle sont ici inversés. Ce n'est plus Julie qui répète poliment les paroles de la comptine, c'est Charlotte qui les écrit et entraîne ainsi son amie dans la danse ; Ce n'est plus Julie qui a faim et soif, c'est Charlotte qui accueille la femme que plus tôt elle regardait intensément. Au travers de la chanson, Charlotte pose un regard extérieur sur son futur couple avec la jeune femme. Le piano maternel, descendu de l'immeuble la séquence précédente, les accompagne et acquiesce à leur amour naissant. On note que les paroles proches de chansons paillardes sont également suggérées par les grands-parents. La maternité inspire Charlotte dans son écriture. Elle écoute le bébé et lui demande conseil. Le ton est enjoué, les paroles humoristiques :

«Elles vécurent ainsi plusieurs années, l'une écrivant et s'occupant du bébé, l'appelant Simon, parfois Simone, l'autre dansant et vivant enfant des rêves de jeunes filles sans amant. L'enfant devenait grand avec ses deux mamans et n'aimait rien que de taper très, très grand sur l'ordinateur ouvert de sa vraie fausse maman des grands mots cochons que lui glissaient doucement ses grands-parents 
resplendissant comme queue, cul, bite, ses grands-parents resplendissant comme queue, cul, bite. L'une notant tout ce que l'enfant disait, ses premiers pas maladroits, ses premiers mots, ses premières dents, ses gazouillements, et ses mamans, l'autre l'oubliant parfois totalement. L'enfant devenait grand avec ses deux mamans et n'aimait rien que de taper très, très grand sur l'ordinateur ouvert de sa vraie fausse maman des grands mots cochons que lui glissaient doucement ses grands-parents resplendissant comme queue, cul, bite, ses grands-parents resplendissants comme queue, cul, comique ».

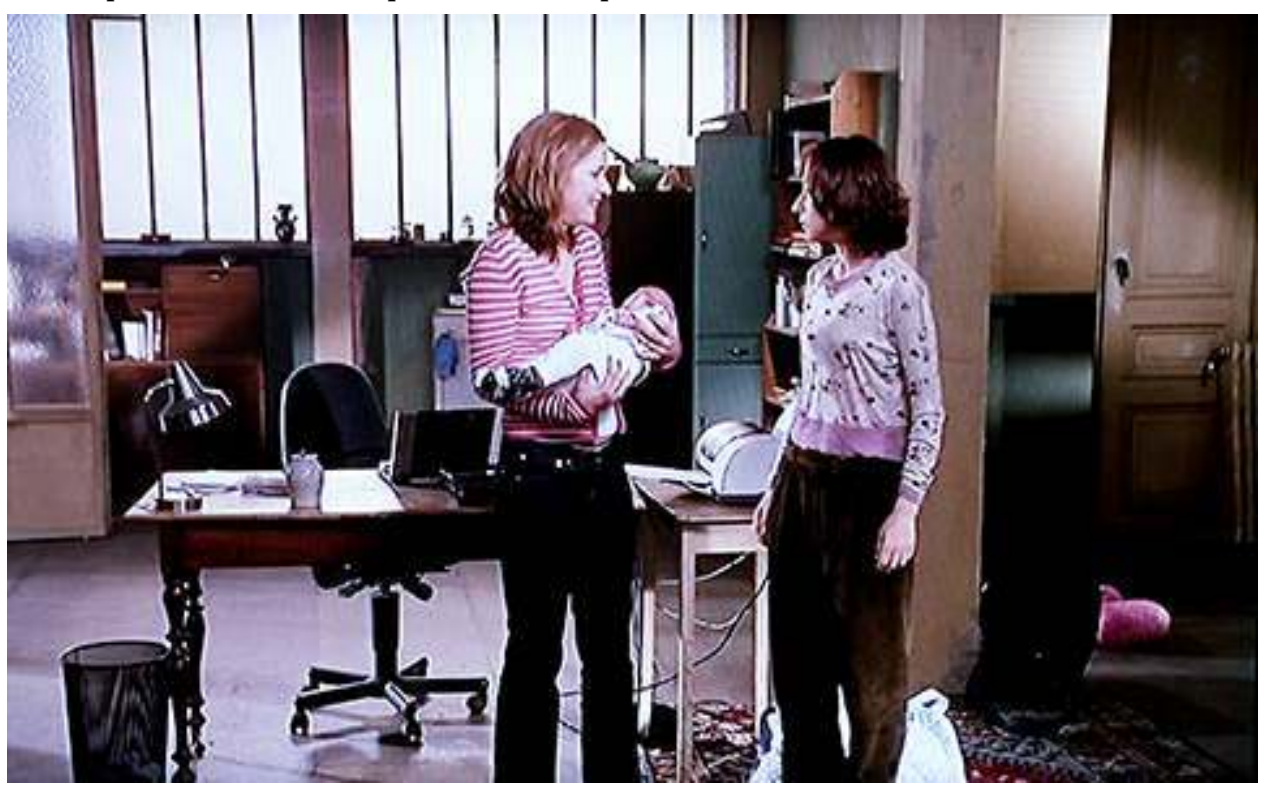

Chantal Akerman, Demain, on déménage, long-métrage, 110 minutes, 2004. rapports dans Demain, on déménage. La relation amoureuse est abordée de manière beaucoup plus légère. L'histoire suggère qu'il suffit simplement d'écouter et de regarder le monde environnant pour éveiller ses sens. Je, tu, il, elle est une prise de conscience, Demain, on déménage est une ouverture vers l'amour. Les deux personnages en témoignent et affirment leurs rencontres en chantant. La danse revient également « l'autre dansant et vivant». Au travers de ces chants, Julie tout comme Charlotte incarnent leur corporalité, vivent enfin le mouvement. Ce n'est pas une musique extérieure à l'action qui accompagne vers le générique mais une déclaration des héroönes du film. Elles bouclent une traversée.

Dans Demain, on déménage, le chant quitte la comptine pour une forme complexe faisant figure d'anachronisme. On remarque en effet un décalage temporel, les paroles sont actuelles alors que la musique fait référence aux chansons populaires engagées des années 1930. Certains éléments du décor sont également issus de cette époque comme la valise, le mobilier et l'aspirateur. À travers ces anachronismes, Chantal Akerman témoigne peut-être de l'intemporalité de l'amour ou en tout cas d'un présent où le passé est toujours là. Charlotte incarne la troisième génération d'après les camps de concentration. Dans cette comédie hors du commun, la cinéaste parvient à ne pas opposer la mémoire d'Auschwitz au bonheur possible d'aujourd'hui. Chanter rappelle ici la tradition juive des chants qui libèrent du passé et permettent d'atteindre un avenir meilleur. Le film agit du côté du présent conscient du passé et allant de l'avant. Le chant accompagne la possibilité de vivre heureux après les traumatismes sans jamais les oublier. À la question posée par Nicole Brenez (de son ami Olivier Wieviorka) sur une 
envie de réaliser un film sur la résistance, Chantal Akerman répond : « Mais j’ai envie de quitter tout ce qui concerne les camps, cela m'a tant emprisonné que j'ai besoin de respirer. Je préfère chanter $»^{21}$ !

\section{La folie Almayer}

14 Le chant de Nina (interprétée par Aurora Marion) dans La folie Almayer ${ }^{22}$ vient confirmer une affirmation grâce au chant de façon plus ambiguë. Ce film onirique, parfois proche du cauchemar, aborde l'émancipation dans la relation entre un père, Gaspard Almayer (interprété par Stanislas Merhar), et sa fille Nina, entre emprise, projection sur l'enfant et désir d'autonomie de celle-ci. S'ajoute à cette dialectique des désirs une dimension identitaire : Nina est la fille d'un homme blanc hollandais et d'une femme malaise. Malgré son métissage, elle reçoit une éducation « de blanche » dans un pensionnat de Malaisie et au retour chez son père, il s'inquiète de son nouveau départ. On ressent le poids de l'exil, du colonialisme, de la passion, l'angoisse de la perte, la folie ruisselante, l'enfermement et la chaleur éprouvante. "Reviens chez ton père, ton père qui t'aime comme un fou » dit Gaspard Almayer. Elle décide de le quitter pour partir avec Dain (interprété par Zac Andrianasolo). Ce n'est pas cet homme qu'elle choisit, sans savoir si elle l'aime vraiment, qui va la sauver, c'est le fait de partir chanter. Elle chante l'Ave verum corpus (1791) de Wolfgang Amadeus Mozart adressé à Jésus : «Ave, ave, verum corpus, natum de maria virgine » ("Je vous salue vrai corps né de la vierge Marie ») et se détache littéralement du décor exotique interprété précédemment par Dain. Sur une scène de dancing, il joue en présonorisation Sway de Dean Martin et Nina, en retrait derrière lui, danse et l'accompagne parmi les choristes. Un homme s'approche et le tue, Nina visiblement dissociée de la réalité continue à danser sur scène jusqu'à ses mots chuchotés : "Nina. Nina. Nina. Nina. Dain est mort.» Elle revient alors au présent, avance vers l'objectif (regard caméra) et en gros plan chante a cappella. Puissance de la lumière, du grain de la peau et de la voix, la combinaison vibratoire tend à nous absorber. Pourtant le choix de l' aria détonne. Alors qu'elle rejette en bloc la culture blanche, Nina choisie un chant chrétien en latin, mais repose la question de l'appartenance, de l'identité et quitte son asservissement par son regard qui tient le nôtre, sans baisser les yeux, dans un face à face égalitaire.

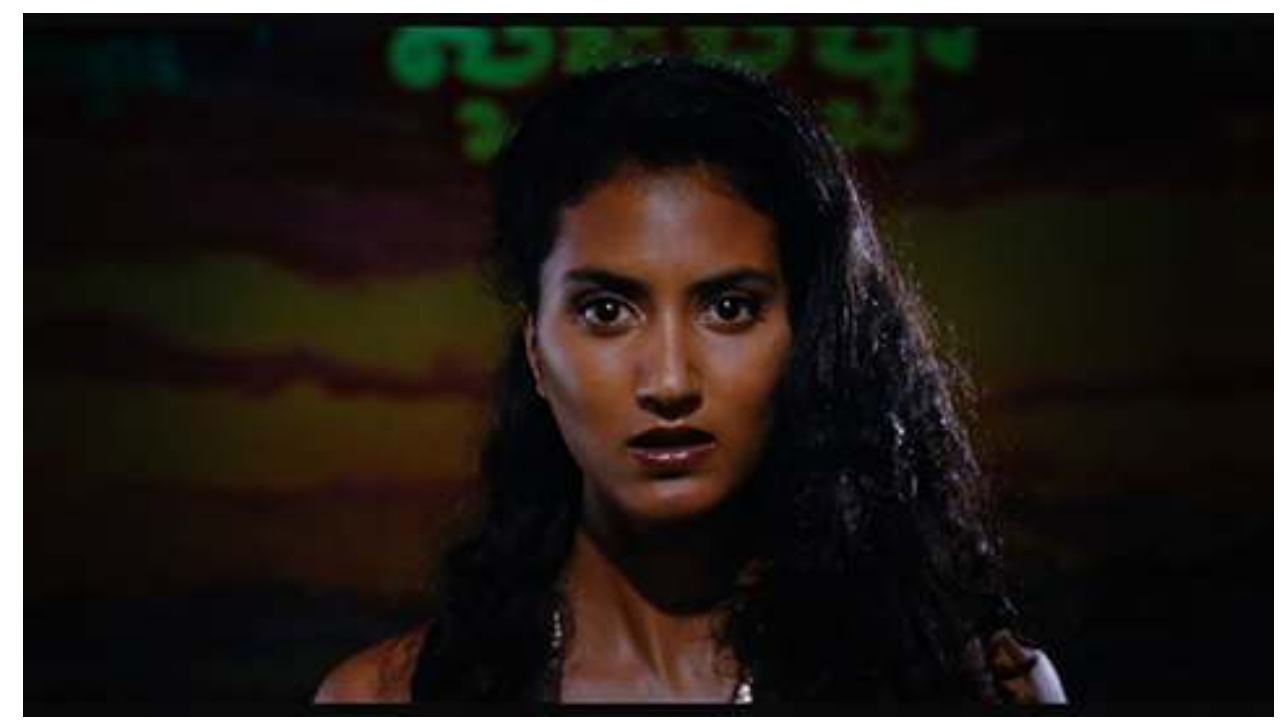

Chantal Akerman, La folie Almayer, long-métrage, 127 min, 2012. 

début du film, mais se situe en fin d'histoire) vers une réception plus sensorielle que narrative du film. Elle raconte : «Je pense que la fille accomplira son destin. Elle chante tout d'un coup dans un boui-boui l'ave verum. Elle chante elle-même, c'est elle qui chante, et tout d'un coup, c'est sans doute par sa voix qu'elle va s'en sortir, par donc son talent, et là on peut en revenir à moi, parce que si je m'en suis sortie c'est parce que j'ai réussi à faire quelque chose, sinon je serais morte $\aleph^{23}$.

Les réalisations de Chantal Akerman, films à voir ou à traverser sous forme d'installation, livre à écouter ou à lire seul, sont parfois qualifiés d'éclectiques. Pourtant un fil traverse son œuvre : une mémoire, un enfermement, une ambivalence face au plaisir et souvent un engagement à s'accomplir coûte que coûte et à faire face à cette forme de prison invisible qui empêche la complétude des personnages et des personnes qu'elle filme. Se dégager de son histoire, de l'Histoire, y échapper, vivre sa vie, là est toute la difficulté. Chanter permet aux personnages de s'affirmer au-delà de leur vulnérabilité. Cette action prolonge la parole et permet de déclarer ce qui peut enfin se dire tout haut, et surtout de passer du dire au faire. En chantant, Julie, Charlotte, Nina, et toutes les autres, sortent du champ, lâchent « prise » et se libèrent.

\section{BIBLIOGRAPHIE}

AKERMAN Chantal, Autoportrait en cinéaste, Chantal Akerman, Éditions du Centre GeorgesPompidou / Éditions Cahiers du cinéma, 2004.

AKERMAN Chantal, Ma mère rit, Édition Mercure de France, Collection Traits et portraits, 2013.

BRENEZ Nicole, Chantal Akerman: The Pajama Interview, Useful Book \#1, Viennale, 2011.

DELEUZE Gilles, Cinéma 2, L'image-temps, Les éditions de minuit, Collection Critique.

\section{Ouvrages collectifs}

DEHAYBE Roger, « Des mots pour une cinéaste » dans Jacqueline Aubenas dir., Hommage à Chantal Akerman, Bruxelles, Édition Roger Dehaybe, 1995.

LEHMAN Boris, « Non éloge (Aujourd'hui je dirais), Des mots pour une cinéaste » dans Jacqueline Aubenas dir., Hommage à Chantal Akerman, Bruxelles, Éditions Roger Dehaybe, 1995.

\section{Articles}

«Chantal Akerman », Atelier des Arts, Cahier n¹, 1982.

LAUFER Laura, Chantal Akerman, Être en résistance, entretien avec Chantal Akerman par LAUFER Laura, revue Rouge, $\mathrm{n}^{\circ} 2203,26$ avril 2007.

PHILIPPON Alain, « Fragments bruxellois, entretien avec Chantal Akerman par Alain Philippon », Cahier du cinéma, nº 341, novembre 1982.

DOLAR Mladen, « Voix », Nous, Grumeaux n¹, mai 2009.

Séquences radiophoniques

Entrelacs, 11 | 2014 
ADLER Laure, entretien avec Chantal Akerman par Laure Adler, Hors-champs, France Culture, 12 janvier 2012.

VEINSTEIN Alain, entretien avec Chantal Akerman par Alain Veinstein, Du jour au lendemain, France Culture, 29 mai 2014.

\section{NOTES}

1. La comédie musicale Golden Eighties (long-métrage, 96 minutes, 1986) est une exception ainsi que Les rendez-vous d'Anna (long-métrage, 127 minutes, 1978) où le chant engendre un nouveau point de vue sur le personnage d'Anna.

2. Chantal Akerman, Je, tu, il, elle, long-métrage, 90 minutes, 1974 (réalisé à partir d'un texte écrit en 1968).

3. Roger Dehaybe, «Des mots pour une cinéaste» dans Jacqueline Aubenas dir., Hommage à Chantal Akerman, Bruxelles, Édition Roger Dehaybe, 1995, p. 49.

4. «Chantal Akerman », Atelier des Arts, Cahier n¹, 1982, p. 155.

5. Chantal Akerman, Ma mère rit, Édition Mercure de France, Collection Traits et portraits, 2013.

6. Chantal Akerman, Portrait d'une paresseuse / Le journal d'une paresseuse, court-métrage, 14 minutes, genre documentaire, 1986.

7. Chantal Akerman, Lettre d'une cinéaste: Chantal Akerman, court-métrage, 8 minutes, Commande pour l'émission Cinéma, cinéma, A2, 1984.

8. Chantal Akerman, Autoportrait en cinéaste, Chantal Akerman, Éditions du Centre GeorgesPompidou / Éditions Cahiers du cinéma, 2004.

9. Alain Veinstein, entretien avec Chantal Akerman par Alain Veinstein, Du jour au lendemain, France Culture, 29 mai 2014.

10. Agnès Varda, Cléo de 5 à 7, long-métrage, 90 minutes, 1962.

11. La durée du film (une heure trente) est quasiment égale à la durée réelle de l'histoire (deux heures).

12. Gilles Deleuze, Cinéma 2, L'image-temps, Les éditions de minuit, Collection critique, p. 255. Alain Philippon, «Fragments bruxellois, entretien avec Chantal Akerman par Alain Philippon », Cahier du cinéma, $\mathrm{n}^{\circ} 341$, novembre 1982, pp. 19-26.

13. Lors d'un entretien avec Alain Philippon, elle explique ses difficultés face à sa technique trop parfaite du cadrage qui entraine une stylisation.

Alain Philippon, « Fragments bruxellois, entretien avec Chantal Akerman par Alain Philippon », Cahier du cinéma, $\mathrm{n}^{\circ} 341$, novembre 1982, pp. 19-26.

14. Laura Laufer, Chantal Akerman, Etre en résistance, entretien avec Chantal Akerman par Laura Laufer, revue Rouge, $\mathrm{n}^{\circ} 2203,26$ avril 2007.

15. Chantal Akerman, Saute ma ville, court-métrage, $11 \mathrm{~min}, 1968$.

16. Mladen Dolar, « Voix », Nous, Grumeaux n¹, mai 2009, p. 17.

17. Jean-Luc Godard, Pierrot le fou, long-métrage, 115 minutes, 1965.

18. Certaines études récentes témoignent de la transformation de l'ADN lors d'un traumatisme sur au moins trois générations lorsqu'il n'est pas soigné.

19. Boris Lehman, «Non éloge (Aujourd'hui je dirais), Des mots pour une cinéaste » dans Jacqueline Aubenas dir., Hommage à Chantal Akerman, Bruxelles, Éditions Roger Dehaybe, 1995, p. 16.

20. Chantal Akerman, Demain, on déménage, long-métrage, 110 minutes, 2004. 
21. «But I want to get away from anything concerning the camps, I've been so caged in by it that I need to breathe. I'd rather sing. " NICOLE BRENEZ, Chantal Akerman: The Pajama Interview, Useful Book \#1, Viennale, 2011.

22. Chantal Akerman, La folie Almayer, long-métrage, $127 \mathrm{~min}, 2012$, (libre adaptation du roman de Joseph Conrad).

23. Laure Adler, entretien avec Chantal Akerman par Laure Adler, Hors-champs, France Culture, 12 janvier 2012.

\section{RÉSUMÉS}

Cette étude propose d'analyser l'apport de la voix chantée dans le cinéma de Chantal Akerman. Dans les films Je, tu, il, elle (1974), Demain on déménage (2004) et La folie Almayer (2012), les personnages quittent la dynamique des chansons de la comédie musicale Golden Eighties (1986) pour chanter une seule fois en fin d'histoire et marquer ainsi une étape essentielle de leur évolution. Grâce au chant, ceux qui se cherchent et se découvrent, s'accomplissent. Ils peuvent enfin se libérer. Au-delà de la dimension physique du chant qui lie cette action au vivre (il faut prendre son souffle pour produire un son vocal), les personnages semblent sortir d'un cadre prédéfini et s'affirmer dans leurs dimensions corporelle et affective.

This study analyses the contribution of the singing voice within Chantal Akerman's cinema. In Je, tu, il, elle (I, you, he, she, 1974), Demain on déménage (Tomorow We Move, 2004) and La folie Almayer ( Almayer's Folly, 2012) the characters leave the songs' dimension of the musical Golden Eighties (1986) to sing only once at the end of the story and thus mark an essential step in their evolution. Thanks to singing, they accomplish themselves and finally feel free. Beyond the physical dimension of singing which ties itself with the action of living (you need to breathe in to produce a vocal sound), characters seem to come out of a predefined frame and to assert themselves in bodily and emotional dimensions.

\section{INDEX}

Mots-clés : Chantal Akerman, Voix, Chant, Comptine, Survivance, émancipation

\section{AUTEUR}

\section{EMMA DUSONG}

Artiste, diplômée de l'ENSBA et chercheuse, docteur en Sciences de l'art et esthétique a présenté sa recherche sur la voix chantée dans le champ de l'art et du cinéma à Paris 1 , à Paris 7, à l'EHESS, à l'Université de Picardie Jules Verne et a mené un cycle de conférences à l'École Nationale Supérieure des beaux-arts de Paris. Elle a également publié des textes dont La compagnie de l'absence, voix chantées et spectralité (revue Volume $\mathrm{n}^{\circ}$ 5, 2012) et Voix mémoires, apparition et disparition dans l'œuvre chantée de Kristin Oppenheim (Observatoire musical français, Université Paris-Sorbonne, dir. Michèle Barbe, à paraître). Son travail artistique et ses films sont présentés 
en France et à l'étranger (Festival International du film de la Rochelle, Festival du court-métrage de Clermont-Ferrand, Lincoln Center, Palais de Tokyo, CRAC Languedoc-Roussillon, Espace culturel Louis Vuitton...). 